\section{IDDF2018-ABS-0156 CLINICAL PROFILE AND FACTORS AFFECTING OUTCOME OF CHILDREN WITH HEPATIC ABSCESS}

Adrienne Michelle Lu. Philippine Pediatric Society, Philippines

\subsection{6/gutjnl-2018-IDDFabstracts.225}

Background Hepatic abscess typically presents with nonspecific symptoms which contribute to late diagnosis and treatment, hence complicated course and outcome. Determining the factors affecting complications and outcome among children with hepatic abscess can help improve the management of these patients. The objective is to determine the clinical profile of children with hepatic abscess and the factors affecting their complications and outcome.

Methods This is a retrospective cohort study. Chart review was done in December 2016 among children 0 to $<19$ years old with a diagnosis of a hepatic abscess on imaging from 1997-2015. Demographic, clinical and diagnostic data gathered were correlated with the outcome and complications.

Results Thirty cases were identified in 19 years, but only 25 charts were reviewed. Mean age in years was 5.27 4.80 SD with male predominance. Fever (96\%) and abdominal pain $(60 \%)$ were common symptoms with Staphylococcus aureus $(56 \%)$ as the most frequent growth on hepatic abscess culture. Anaemia (76\%) and leukocytosis (96\%), as well as solitary (76\%), large abscesses $>5 \mathrm{cms}$ (60\%) involving the right lobe (72\%), were also common. Most were treated with antibiotics alone (60\%). All patients improved without mortalities, while pleural effusion (67\%) was a commonly seen complication. Only male gender was significantly associated with complications both on Chi-square ( $p$ 0.004) and logistic regression ( $p$ 0.008).

Conclusions Hepatic abscess is common among the young and male population, manifesting as fever with anaemia and leukocytosis, and Staphylococcus aureus commonly growing on hepatic abscess culture. Most were complicated by pleural effusion with no deaths reported. Male gender had a significant association with complications.

\section{IDDF2018-ABS-0168 UTILITY OF FDG-PET/CT IN PRE- TRANSPLANTATION WORKUP OF THE POTENTIAL LIVER RECIPIENT WITH HEPATOCELLULAR CARCINOMA}

\footnotetext{
${ }^{1}$ Sang young $\mathrm{Yi}^{*}$, ${ }^{1} \mathrm{Ju}$ Hyun Shim, ${ }^{2} \mathrm{Gi}$ Won Song, ${ }^{1} \mathrm{G}$ wang Hyeon Choi, ${ }^{1}$ Kang Mo Kim, ${ }^{1}$ Young-Suk Lim, 'Han Chu Lee, ${ }^{1}$ Young-Hwa Chung, ${ }^{1}$ Yung Sang Lee. 'Department of Gastroenterology, Asan Medical center, University of Ulsan College of Medicine, Korea, South; ${ }^{2}$ Department of Surgery, Asan Medical center, University of Ulsan College of Medicine, Korea, South
}

\subsection{6/gutjnl-2018-IDDFabstracts.226}

Background There is no consensus approach to the decisionmaking regarding suitability for liver transplantation (LT) in patients with hepatocellular carcinoma (HCC). Positron emission tomography-computed tomography using ${ }^{18} \mathrm{~F}$-fluorodeoxyglucose (FDG PET/CT) has been found to provide helpful information for determining post-LT prognosis in HCC patients. We aimed to assess the performance of FDG-PET/CT for detection of metastatic disease or other malignancy in potential liver recipients with HCC.

Methods This retrospective study included 423 adult liver transplant candidates with HCC who underwent both FDGPET/CT and conventional imaging studies such as chest-abdomen-pelvis CT examinations and bone scintigraphy, together with upper and lower gastrointestinal endoscopy, as pre-transplant workup. The diagnostic performance of FDG-PET/CT was quantitatively evaluated by the calculation of sensitivity, specificity, positive predictive value (PPV), and negative predictive value (NPV).

Results Out of the 423 patients, 338 (79.9\%) were male with a median age of 55 years (54.03-55.43). In terms of extrahepatic evaluation, FDG-PET/CT detected a total of 163 lesions in 142 patients, which consisted of 132 benign and 31 malignant-looking lesions. The number of lesions suspicious for metastatic HCC was initially 24. Among the 17 metastases, 4 were suspected to be a metastatic focus only by FDG-PET/CT, not by conventional scanning. All 2 cases of colorectal cancers diagnosed by sigmoidoscopic screening were detected on neither FDG-PET/CT nor abdomen/pelvis CT images. False-positive FDG-PET/CT results were identified in 12 patients with benign lesions. No false negative errors occurred in FDG-PET/ CT, while $1.2 \%$ in conventional studies. Taken together, the performance of FDG-PET/CT in detecting metastatic or primary cancer in our series was measured with a sensitivity of $90.48 \% / 89.47 \%$, specificity of $97.04 \% / 97.04 \%, \quad \mathrm{PPV}$ of $61.29 \% / 62.96 \%$, and NPV of $99.49 \% / 99.49 \%$ on per-lesion/ per-patient basis.

Conclusions Our results indicate that whole-body FDG-PET/ CT, when coupled with the colonic examination, is effective for identification of extrahepatic disorders before LT in patients with HCC and may fully replace multiple organ-specific imaging tests.

\section{IDDF2018-ABS-0173 A STUDY ON OUTCOME OF HEPATITIS C PATIENTS IN A COMMUNITY WITH PREDOMINANT GENOTYPE 3 WITH STANDARD OF CARE TREATMENT BEFORE AND AFTER ADVENT OF DIRECT ACTING ANTIVIRALS IN KASHMIR VALLEY A RETROSPECTIVE CUM PROSPECTIVE STUDY}

Nisar Shah*. Government Medical college Srinagar JandK (India), India

\subsection{6/gutjnl-2018-IDDFabstracts.227}

Background Hepatitis C virus (HCV) was first identified in 1989 and was the major cause of non-A, non-B viral hepatitis. $\mathrm{HCV}$ is a globally prevalent pathogen and is a major cause of healthcare burden in India. As per current estimates, more than 165 million people around the world are infected with $\mathrm{HCV}$, of whom 350000 die each year. The treatment of hepatitis $\mathrm{c}$ has evolved by leaps and bounds in last two decades to almost a perfection at present. The aim of our study was to study and compare the response to different regimen during and after the Interferon era.

Methods The current study was a hospital-based prospective cum retrospective study. For retrospective data, 200 Hepatitis 'C' patients (Genotype 3) from 2011 to 2014 were analysed with respect to patients' demography, Quantitative Hepatitis 